\title{
Cost-effectiveness of manual therapy versus physiotherapy in patients with sub-acute and chronic neck pain: a randomised controlled trial
}

\author{
J. M. van Dongen ${ }^{1} \cdot$ R. Groeneweg ${ }^{1,2,3} \cdot$ S. M. Rubinstein ${ }^{1} \cdot$ J. E. Bosmans ${ }^{1}$ • \\ R. A. B. Oostendorp ${ }^{2,4} \cdot$ R. W. J. G. Ostelo ${ }^{1,5} \cdot$ M. W. van Tulder ${ }^{1,5}$
}

Received: 16 November 2015/Revised: 8 March 2016/Accepted: 10 March 2016/Published online: 21 March 2016

(C) The Author(s) 2016. This article is published with open access at Springerlink.com

\begin{abstract}
Purpose To evaluate the cost-effectiveness of manual therapy according to the Utrecht School (MTU) in comparison with physiotherapy (PT) in sub-acute and chronic non-specific neck pain patients from a societal perspective. Methods An economic evaluation was conducted alongside a 52-week randomized controlled trial, in which 90 patients were randomized to the MTU group and 91 to the PT group. Clinical outcomes included perceived recovery (yes/no), functional status (continuous and yes/no), and quality-adjusted life-years (QALYs). Costs were measured from a societal perspective using self-reported questionnaires. Missing data were imputed using multiple imputation. To estimate statistical uncertainty, bootstrapping techniques were used.
\end{abstract}

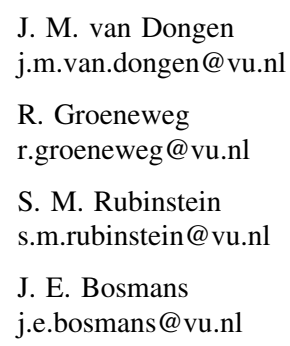

1 Department of Health Sciences \& EMGO+ Institute for Health and Care Research, Faculty of Earth \& Life Sciences, VU University Amsterdam, De Boelelaan 1085,

1081 HV Amsterdam, The Netherlands
Results After 52 weeks, there were no significant between-group differences in clinical outcomes. During follow-up, intervention costs $(\beta: €-32 ; 95 \% \mathrm{CI}:-54$ to $-10)$ and healthcare costs $(\beta: €-126 ; 95 \% \mathrm{CI}:-235$ to -32 ) were significantly lower in the MTU group than in the PT group, whereas unpaid productivity costs were significantly higher ( $\beta: € 186 ; 95 \% \mathrm{CI}: 19-557)$. Societal costs did not significantly differ between groups $(\beta: €-96$; $95 \%$ CI:-1975-2022). For QALYs and functional status (yes/no), the maximum probability of MTU being costeffective in comparison with PT was low $(\leq 0.54)$. For perceived recovery (yes/no) and functional status (continuous), a large amount of money must be paid per additional unit of effect to reach a reasonable probability of costeffectiveness.

2 Scientific Institute for Quality of Health Care, Radboud University Nijmegen Medical Centre, Geert Grooteplein 21, 6525 EZ Nijmegen, The Netherlands

3 Avansplus, University for Professionals, Heerbaan 14-40, 4817 NL Breda, The Netherlands

4 Department of Manual Therapy, Faculty of Medicine and Pharmacy, Free University of Brussels, Pleinlaan 2, 1050 Brussels, Belgium

5 Department of Epidemiology and Biostatistics \& the EMGO+ Institute for Health and Care Research, VU University Medical Center, De Boelelaan 1089a, 1081 HV Amsterdam, The Netherlands 
Conclusions From a societal perspective, MTU was not cost-effective in comparison with PT in patients with subacute and chronic non-specific neck pain for perceived recovery, functional status, and QALYs. As no clear total societal cost and effect differences were found between MTU and PT, the decision about what intervention to administer, reimburse, and/or implement can be based on the preferences of the patient and the decision-maker at hand.

Trial registration ClinicalTrials.gov Identifier: NCT00713843

Keywords Economic evaluation - Musculoskeletal manipulation $\cdot$ Physical therapy modalities

\section{Introduction}

Neck pain is a major public health problem in Western countries, with two-thirds of the population experiencing neck pain at some stage in their life [1]. Neck pain has serious consequences for the patients' personal health and overall well-being, can severely hinder daily activity and participation, and has large economic consequences for society [2]. To illustrate the latter, the annual cost of neck pain to Dutch society was estimated at \$668 million in 1996 [3].

Conservative treatments for neck pain include care by a general practitioner (GP; e.g., advice, analgesics), manual therapy (e.g., manipulation, mobilisation), physiotherapy (PT; e.g., exercise therapy, graded activity program), and combinations thereof [4]. In the Netherlands, most neck pain patients either receive care by a GP or treatment by physiotherapists. The latter mainly deliver exercise therapy combined with advice and instructions for physical activities, sometimes supplemented with manual therapy [5]. Korthals-de Bos et al. (2003) found manual therapy to be cost-effective compared with GP care and PT in sub-acute and chronic neck pain patients [6]. A more recent RCT, however, indicated that manual therapy was neither effective nor cost-effective compared with behavioural graded activity in sub-acute neck pain patients [7].

In the Netherlands, various forms of manual therapy exist, of which "Manual Therapy according to the Utrecht School" (MTU) has not yet been rigorously evaluated. MTU is characterized by specific diagnostic and treatment techniques, but also incorporates techniques that are frequently used in other manual therapies for neck pain, such as stabilisation and joint mobilisation. In a recent RCT, MTU (consisting of joint mobilisation) and PT (consisting of exercise therapy, including instructions and advice) were found to be equally effective for perceived recovery, functional status, and pain among sub-acute and chronic non-specific neck pain patients, while fewer treatments were found to be needed for MTU [8]. It is unknown, however, whether the latter translates into societal cost savings and whether MTU is cost-effective compared with PT. Therefore, the present study aimed to evaluate the societal cost-effectiveness of MTU in comparison with PT in sub-acute and chronic non-specific neck pain patients.

\section{Methods}

\section{Design}

An economic evaluation was conducted alongside a Dutch 52-week pragmatic RCT [8]. The study design and informed consent procedure were approved by the Medical Ethics committee CMO Arnhem-Nijmegen (NL21128.091.08).

Patients could enter the study by directly presenting at one of the participating practices or through GP referral. Additionally, participants were recruited through advertisements in local newspapers [8]. Upon entering the trial, participants provided informed consent and a research assistant performed the baseline measurements. Subsequently, participants were randomised to the MTU or PT group by an independent research assistant using a central computer generated randomisation scheme. Randomisation was performed at the individual level using block randomization $($ size $=4)$, with pre-stratification for pain (range: 0 (no pain) to 10 (maximum pain); $<7$ versus $\geq 7$ ) and age ( $<40$ versus $\geq 40$ years) [8].

\section{Participants}

Sixteen primary healthcare centres for manual therapy and PT participated in the study (i.e., patients were recruited and treated in all of these centres). Centres employed at least one manual therapist, one physiotherapist, and one research assistant. Adults (18-70 years) who had neck pain for more than 2 weeks, but no longer than 52 weeks were eligible for inclusion. Neck pain was defined as "pain in the cervical region with or without radiation to the shoulder region or upper extremities, and/or accompanied by headaches as long as the main pain complaint was the neck". Exclusion criteria were: red flags; previous cervical surgery; pregnancy; whiplash; conditions that seriously impede treatment; insufficient knowledge of the Dutch language; or conservative neck pain treatment during the previous 3 months.

\section{Interventions}

\section{Manual Therapy according to the Utrecht school}

During the first MTU session, manual therapists performed a general assessment of the participant, including medical 
history, signs and symptoms, red flags, and treatment indication. Additionally, specific tests were conducted to evaluate the participants' movement preferences. During treatment, these preferred movements were passively executed by the manual therapist in a combination of rolling and sliding, or rocking and gliding, in the joints of the spine and extremities. In MTU, it is also common to give advice on physical activities and lifestyle, and to recommend exercise. MTU sessions were scheduled once every 1-2 weeks, with a maximum of six sessions, and lasted 30-60 min. Per patient, the number of sessions was determined by the manual therapist and depended on the patient's condition and/or progression. Manual therapists followed a 3-year post-graduate training at the School of Manual Therapy Utrecht and had a minimum working experience of 5 years [8].

\section{Physiotherapy}

During the first PT session, physiotherapists performed a general assessment of the participant, including medical history, signs and symptoms, red flags, and treatment indication, after which a treatment plan and treatment goals were established. Treatment could consist of active exercises, muscle stretching, manual traction, and massage. During each treatment session, physiotherapists spent at least $20 \mathrm{~min}$ on active exercises combined with instruction. Specific manual techniques (i.e., mobilization, manipulation) were not performed. Physiotherapy sessions took place $\leq 2$ times a week, with a maximum of nine sessions, and lasted about $30 \mathrm{~min}$. Per patient, the number of sessions was determined by the physiotherapist and depended on the patient's condition and/or progression. Physiotherapists followed a 4-year training in PT and did not have an educational background in manual therapy.

\section{Clinical measures}

Baseline measurements included participant characteristics, demographic variables, and potential confounding variables.

Perceived recovery (yes/no) and functional status were assessed at 3, 7, 13, 26, 39, and 52 weeks using the global perceived effect (GPE) and Neck Disability Index-Dutch Version (NDI-DV), respectively. The GPE measures a participant's subjective global improvement using a 7-point scale ranging from "worse than ever"(1) to "completely recovered"(7) [9]. Perceived recovery (yes) was defined as being "completely recovered" or "much improved"; other responses were defined as not recovered (no). The NDI-DV measures a participant's self-rated physical disability. The NDI-DV score ranges from 0 to 50, with higher scores indicating higher disability levels. The proportion of responders on the NDI-DV was also estimated. Response was defined as improving $\geq 4$ points on the NDI-DV (yes); other responses were defined as not responded (no) [10].

Health-related quality of life was assessed at baseline, 7, and 52 weeks using the SF-6D. The participants' SF-6D health states were translated into utility scores using the UK tariff [11]. Quality-adjusted life years (QALYs) were calculated by multiplying the participants' utility scores by their time spent in a health state using linear interpolation between measurement points.

\section{Cost measures}

Costs included intervention, healthcare, informal care, absenteeism, and unpaid productivity costs due to neck pain. Cost measures were assessed at 3, 7, 13, 26, 39, and 52 weeks using self-reported questionnaires. All costs were converted to Euros 2010 using consumer price indices [12]. Discounting of costs was not necessary due to the 52-week follow-up [13].

Intervention costs were estimated by determining the participants' total number of MTU or PT sessions during the intervention period, and valuing them using Dutch standard costs [14].

Healthcare utilization included care by a primary and secondary healthcare provider and the use of prescribed and over-the-counter medication. Primary and secondary healthcare utilization were valued using Dutch standard costs and prices according to professional organizations [14]. Medication use was valued using unit prices of the Royal Dutch Society of Pharmacy [15].

Informal care (i.e., care by family, friends, and other volunteers) was valued using a shadow price of $€ 12.7 / \mathrm{h}$ [14].

Absenteeism was assessed by asking participants to report their total number of sickness absence days due to neck pain. Using the Friction Cost Approach (FCA), absenteeism was valued using age- and gender-specific price weights ${ }^{36}$. The FCA assumes that costs are limited to the friction period (i.e., period needed to replace a sick worker $=23$ weeks) [14].

Unpaid productivity losses (i.e., volunteer work, and domestic and educational activities that participants are not able to perform) were valued using a shadow price of $€ 12.7 / \mathrm{h}[14]$.

\section{Data analysis}

Intention-to-treat analyses were performed. Descriptive statistics were used to compare baseline characteristics between MTU and PT group participants, and those with 
complete and incomplete data. Missing data were multiply imputed, stratified by treatment group. Using fully conditional specification and predictive mean matching, 15 datasets were created in IBM SPSS (v22, Chicago, IL) (loss-of-efficiency $<5 \%$ ) [16]. Each dataset was analysed separately as specified below. Pooled estimates were calculated using Rubin's rules, incorporating both withinimputation variability (i.e., uncertainty about the results from one imputed data set) and between-imputation variability (i.e., reflecting the uncertainty due to the missing information) [16].

Effectiveness at 52 weeks was estimated using linear regression analyses, adjusted for baseline values. To compare costs between groups, linear regression analyses were performed as well. Seemingly unrelated regression (SUR) analyses were performed to estimate total cost and effect differences (i.e., $\Delta \mathrm{C}$ and $\Delta \mathrm{E}$ ). An advantage of SUR is that two regression equations (i.e., one for $\Delta \mathrm{C}$ and one $\Delta \mathrm{E})$ are modelled simultaneously so that their possible correlation can be accounted for [17]. Incremental costeffectiveness ratios (ICERs) were calculated by dividing the corrected difference in total costs by that in effects (i.e., $\Delta \mathrm{C} / \Delta \mathrm{E})$. Uncertainty surrounding the ICERs and $95 \%$ confidence intervals (95\%CIs) around cost differences were estimated using bias corrected and accelerated (BCA) bootstrapping (5000 replications). Uncertainty was graphically illustrated by plotting bootstrapped incremental costeffect pairs (CE-pairs) on cost-effectiveness planes (CEplanes) [13]. A summary measure of the joint uncertainty of costs and effects was provided using cost-effectiveness acceptability curves (CEACs). Such curves provide an indication of the probability of MTU being cost-effective in comparison with PT at different values of willingnessto-pay [13]. Unless otherwise stated, data were analysed in STATA (V12, Stata Corp, TX). Statistical significance was set at $p<0.05$.

\section{Sensitivity analyses}

Three sensitivity analyses were performed. In a first sensitivity analysis (SA1), only data of complete cases were included. In a second sensitivity analysis (SA2), absenteeism costs were estimated using the Human Capital Approach, assuming that productivity losses are generated during the entire duration of absence. In a third sensitivity analysis (SA3), QALYs were estimated using the EQ-5D, which was administered at 3, 7, 13, 26, 39, and 52 weeks, but not at baseline. Utility scores were estimated using the Dutch tariff [18]. Under the premise that the participants' health states did not change between baseline and 3-week follow-up, QALYs were calculated using linear interpolation between measurement points.

\section{Results}

\section{Participants}

One hundred and eighty one sub-acute and chronic nonspecific neck pain patients participated. Of them, 90 were randomized to the MTU group and 91 to the PT group (Fig. 1). At baseline, no relevant differences were found between groups (Table 1). Complete data were obtained from 114 participants $(62 \%)$ on the effect measures and 147 participants $(81 \%)$ on the cost measures. Some significant differences were observed between participants with complete and incomplete data in both treatment groups (Table 1). These variables were included in the imputation model.

\section{Effects}

No significant differences were found between the MTU and PT group in perceived recovery (yes/no: $\beta=0.09$; $95 \%$ CI: $-0.05-0.24$ ), functional status (continuous: $\beta=-1.03 ; 95 \%$ CI: $-2.55-0.48$, yes $/$ no: $\beta=-0.01$; $95 \% \mathrm{CI}:-0.15-0.13)$, and QALYs $(\beta=-0.01$; $95 \% \mathrm{CI}$ : $-0.04-0.03)$.

\section{Resource use and costs}

Participants in the MTU group visited a manual therapist on average 3.0 times, while participants in the PT group visited a physiotherapist on average 5.1 times. This resulted in a significant difference in intervention costs between both groups in favour of the MTU group. MTU group participants had significantly lower healthcare costs and significantly higher unpaid productivity costs than their PT group counterparts. All other between-group differences were not significant (Table 2).

\section{Cost-effectiveness}

The ICER for perceived recovery was -1024 , indicating that an additional recovered patient in the MTU group compared with the PT group was associated with a societal cost saving of $€ 1024$ (Table 3; Fig. 2-1a). The CEAC in Fig. 2-2a indicates that the maximum probability of costeffectiveness was 0.88 . To reach this probability, societal decision-makers should be willing to pay $€ 39,000$ per additional recovered patient in the MTU group compared with the PT group.

When functional status was evaluated as a continuous outcome, an ICER of 92 was found. This indicates that an additional 1-point improvement on the NDI-DV was associated with a societal cost saving of $€ 92$ (Table 3; Fig. 2-1b). The CEAC presented in Fig. 2-2b indicates that 


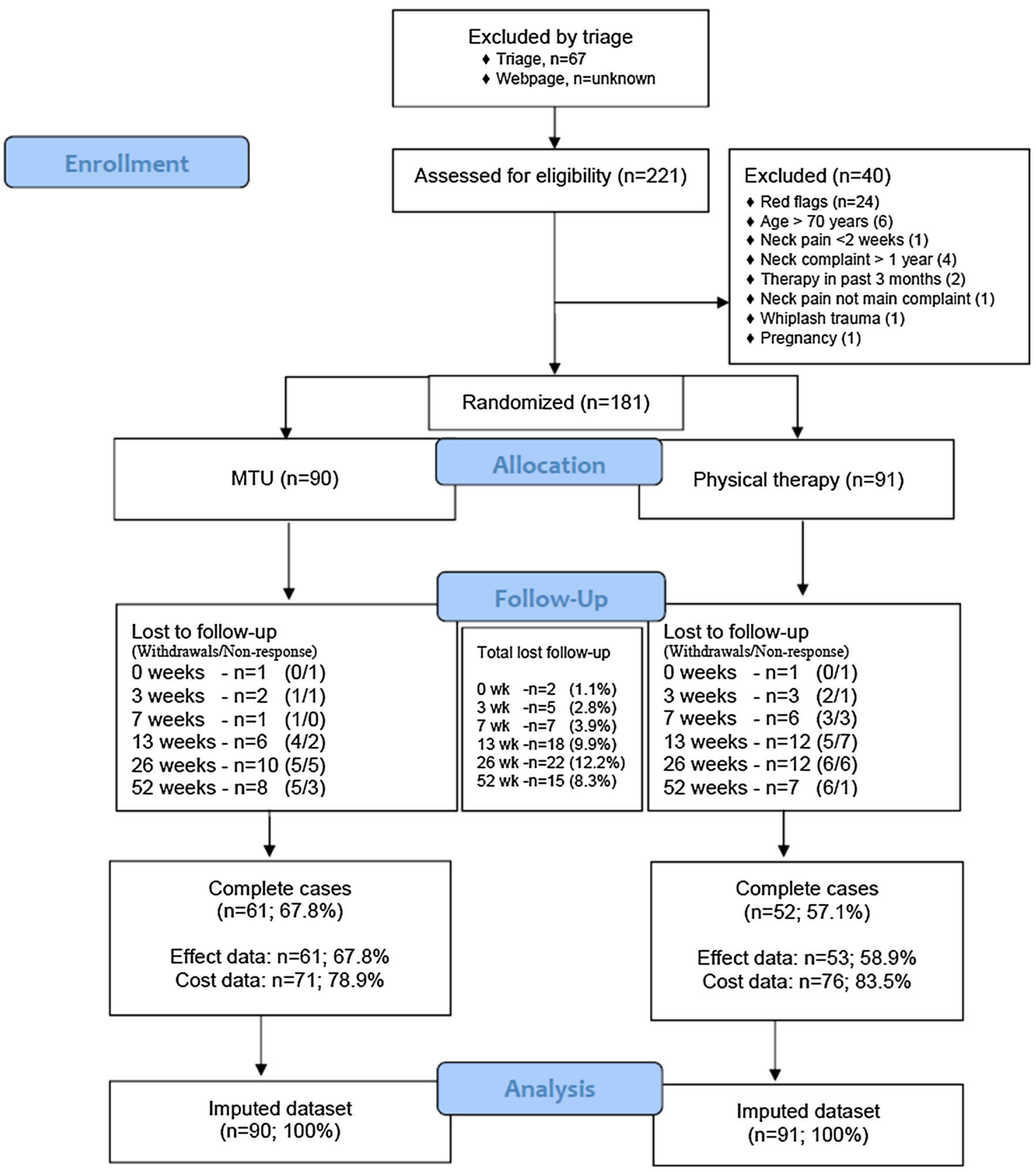

Fig. 1 Flowchart of trial participants

the maximum probability of cost-effectiveness was 0.91 if societal decision-makers are willing to pay $€ 4000$ per additional 1-point improvement on the NDI-DV in the MTU group compared with the PT group.

When functional status was evaluated as a dichotomous outcome (yes/no), an ICER of 7314 was found. This indicates that $€ 7314$ was saved by society per participant less with an improvement of $\geq 4$ points on the NDI-DV (Table 3). The CEAC (not shown) indicated that the maximum probability of MTU being cost-effective compared with PT was low $(\leq 0.54)$, irrespective of the willingness-to-pay.

For QALYs, an ICER of 14,561 was found, indicating that one QALY lost was associated with a societal cost saving of $€ 14,561$ (Table 3). The CEAC (not shown) indicated that the maximum probability of MTU being cost-effective in comparison with PT was low $(\leq 0.54)$, irrespective of the willingness-to-pay. 
Table 1 Baseline characteristics of Manual Therapy Utrecht (MTU) group and Physiotherapy (PT) group participants

\begin{tabular}{|c|c|c|c|c|c|c|c|c|}
\hline \multirow[t]{2}{*}{ Baseline characteristics } & \multicolumn{3}{|l|}{ MTU group } & \multirow[b]{2}{*}{$P$ value } & \multicolumn{3}{|l|}{ PT group } & \multirow[b]{2}{*}{$P$ value } \\
\hline & $\begin{array}{l}\text { All } \\
(n=90)\end{array}$ & $\begin{array}{l}\text { Complete } \\
(n=61)\end{array}$ & $\begin{array}{l}\text { Incomplete } \\
(n=29)\end{array}$ & & $\begin{array}{l}\text { All } \\
(n=91)\end{array}$ & $\begin{array}{l}\text { Complete } \\
(n=52)\end{array}$ & $\begin{array}{l}\text { Incomplete } \\
(n=39)\end{array}$ & \\
\hline Female $[\mathrm{n}(\%)]$ & $56(62.2)$ & $39(63.9)$ & $17(58.6)$ & 0.627 & $56(61.5)$ & $25(48.7)$ & $31(79.5)$ & 0.002 \\
\hline Age (years) [mean (SD)] & $49.2(12.4)$ & $47.3(12.8)$ & $53.3(10.8)$ & 0.030 & $48.7(12.6)$ & $49.5(13.0)$ & $47.8(12.2)$ & 0.529 \\
\hline First neck pain episode $[n(\%)]$ & $56(62.2)$ & $38(62.3)$ & $18(62.1)$ & 0.767 & $58(63.7)$ & $33(63.5)$ & $25(64.1)$ & 0.950 \\
\hline \multicolumn{9}{|l|}{ Main complaint $[n(\%)]$} \\
\hline Pain & $79(87.8)$ & $55(90.2)$ & $24(82.8)$ & 0.316 & $80(87.9)$ & $44(84.6)$ & $36(92.3)$ & 0.265 \\
\hline Stiffness & $30(33.3)$ & $20(32.8)$ & $10(25.6)$ & 0.873 & $38(41.8)$ & $20(38.5)$ & $18(46.2)$ & 0.007 \\
\hline Mobility impairment & $33(36.7)$ & $17(27.9)$ & $16(55.2)$ & 0.005 & $37(40.7)$ & $18(34.6)$ & $19(48.7)$ & 0.175 \\
\hline Other & $7(7.8)$ & $1(1.6)$ & $6(20.7)$ & 0.002 & $10(11.0)$ & $8(15.4)$ & $2(5.1)$ & 0.122 \\
\hline $\begin{array}{l}\text { Complaint intensity (NRS: } \\
0-10) \text { [mean (SD)] }\end{array}$ & $5.5(2.0)$ & $5.4(2.1)$ & $5.7(2.0)$ & 0.531 & $5.8(1.8)$ & $5.7(1.6)$ & $6.1(2.1)$ & 0.296 \\
\hline $\begin{array}{l}\text { Functional status (NDI-DV: } \\
0-50) \text { [mean (SD)] }\end{array}$ & $12.5(6.8)$ & $11.6(5.6)$ & $14.5(8.7)$ & 0.061 & $11.7(5.4)$ & $11.0(4.5)$ & $12.6(6.3)$ & 0.157 \\
\hline Utility score $(0-1)$ [mean $(\mathrm{SD})]$ & $0.71(0.13)$ & $0.73(0.11)$ & $0.65(0.14)$ & 0.009 & $0.71(0.12)$ & $0.72(0.11)$ & $0.69(0.12)$ & 0.148 \\
\hline
\end{tabular}

$n$ number, $S D$ standard deviation, $N R S$ numeric rating scale, $N D I-D V$ neck disability index-Dutch version

Table 2 Mean costs per participant in the Manual Therapy Utrecht (MTU) group and Physiotherapy (PT) group, and mean cost differences between both groups during follow-up

\begin{tabular}{lccc}
\hline Cost category & $\begin{array}{l}\text { MTU group } \\
n=90 ; \text { mean (SEM) }\end{array}$ & $\begin{array}{l}\text { PT group } \\
n=91 ; \text { mean (SEM) }\end{array}$ & $\begin{array}{l}\text { Mean cost difference } \\
\text { (95\% CI) }\end{array}$ \\
\hline Intervention $^{\text {a }}$ & $114(6)$ & $145(9)$ & $-32(-54$ to -10$)$ \\
Healthcare $^{\mathrm{a}}$ & $172(28)$ & $298(43)$ & $-126(-235$ to -32$)$ \\
Primary healthcare & $117(18)$ & $226(34)$ & $-109(-207$ to 45$)$ \\
Secondary healthcare & $48(17)$ & $58(17)$ & $-10(-55$ to 41$)$ \\
Medication & $7(3)$ & $14(5)$ & $-7(-23$ to 1$)$ \\
Informal care & $106(64)$ & $62(22)$ & $43(-36$ to 310$)$ \\
Absenteeism & $1702(726)$ & $1870(656)$ & $-168(-1994$ to 1810$)$ \\
Unpaid productivity & $348(115)$ & $162(36)$ & $186(19$ to 557$)$ \\
Total & $2442(758)$ & $2537(681)$ & $-96(-1975$ to 2022) \\
\hline
\end{tabular}

$n$ number, SEM standard error of the mean, $C I$ confidence interval, Note costs are expressed in 2010 Euros

${ }^{a}$ Healthcare costs are the sum of the primary healthcare costs, secondary healthcare costs, and medication costs

\section{Sensitivity analyses}

In line with the main analysis, between-group differences in total costs and effects were not significant in all sensitivity analyses. The overall conclusion of this study would not change when using any of the assumptions of the sensitivity analyses.

\section{Discussion}

\section{Main findings}

In comparison with PT, MTU was associated with significantly lower intervention costs and healthcare costs, whereas unpaid productivity costs were significantly higher. The latter was due to the fact that more MTU group patients reported to experience unpaid productivity losses compared with their PT group counterparts $\left(X^{2}=18.5\right.$; $p=0.000$ ). Total costs did not significantly differ between groups, nor did all other between-group differences in costs and effects. For QALYs and functional status (yes/no), the maximum probability of MTU being cost-effective in comparison with PT was low $(\leq 0.54)$. For perceived recovery and functional status (continuous), large amounts of money must be paid by society per additional unit of effect to reach a reasonable probability of cost-effectiveness (e.g., $€ 39,000$ per additional recovered patient for a probability of 0.88 ). Sensitivity analyses confirmed these results. Therefore, MTU cannot be regarded as cost-effective in comparison with PT from a societal perspective. As no clear total societal cost and effect differences were 


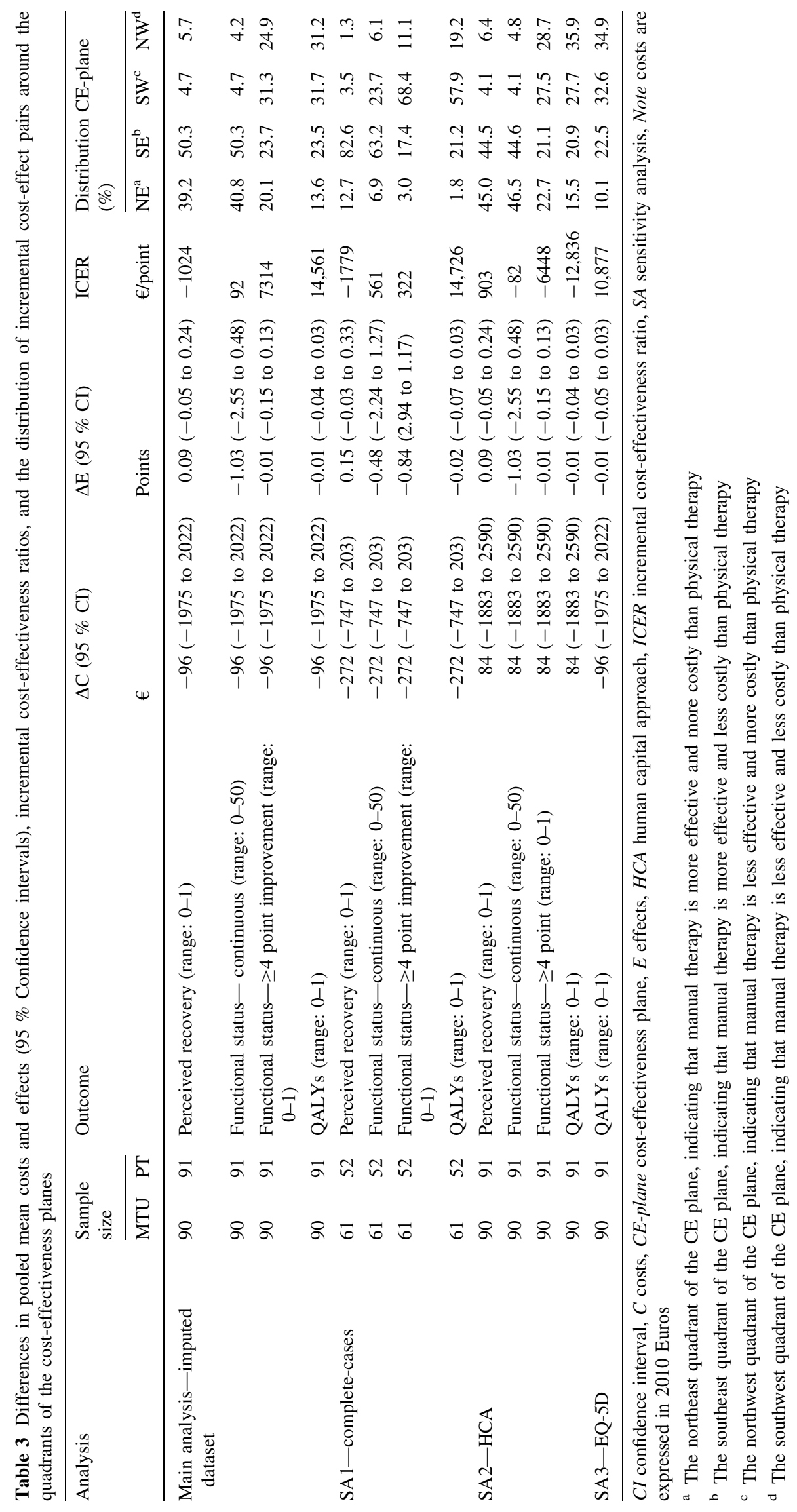




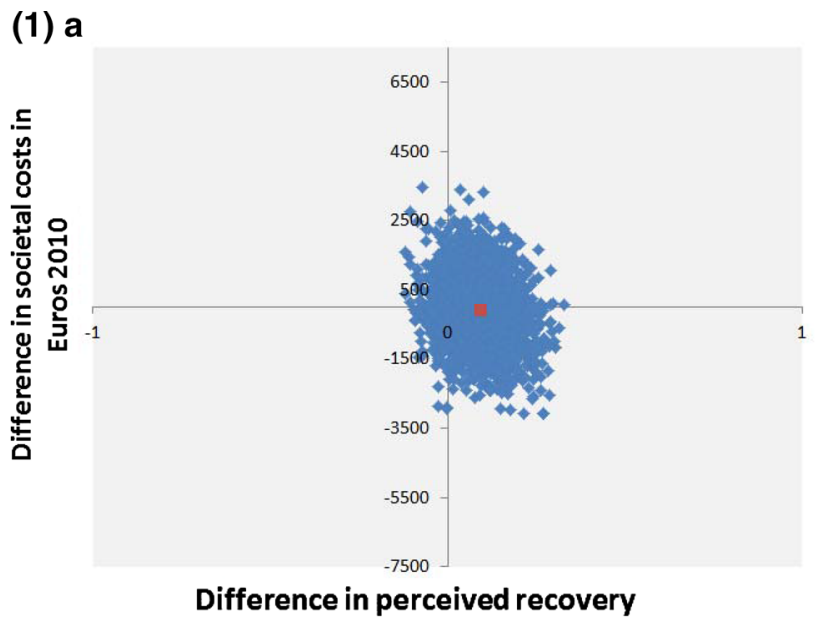

(1) b

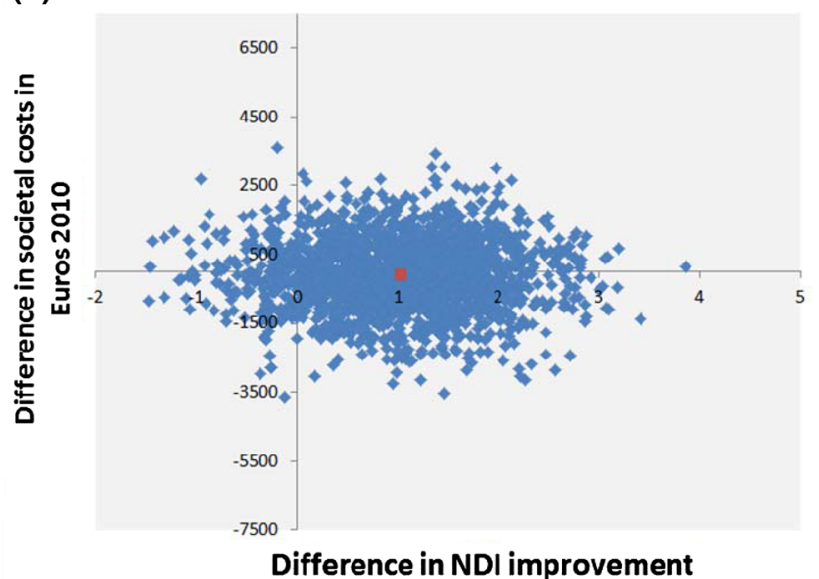

Fig. 2 Cost-effectiveness planes indicating the distribution of incremental cost-effect pairs around its four quadrants (1) and costeffectiveness acceptability curves indicating the probability of manual

found between MTU and PT, the decision about what intervention to administer, reimburse, and/or implement can be based on the preferences of the patient and the decision-maker at hand.

\section{Interpretation of the findings}

There may be several potential explanations for the finding that MTU was not cost-effective in comparison with PT. First, only one form of manual therapy (i.e., MTU) was compared to PT, whereas a more eclectic approach to manual therapy may be more likely to be (cost-)effective. This explanation is underscored by the study of Korthals-de Bos et al. (2003) who found manual therapy to be cost-effective in comparison with PT when it incorporated several techniques used in Western Europe, North America, and Australia, including those
(2) a

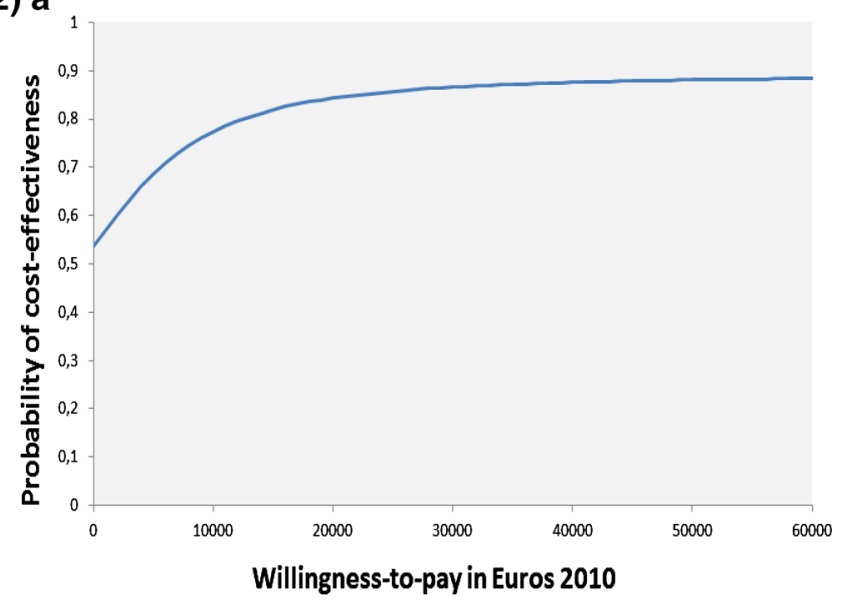

(2) b

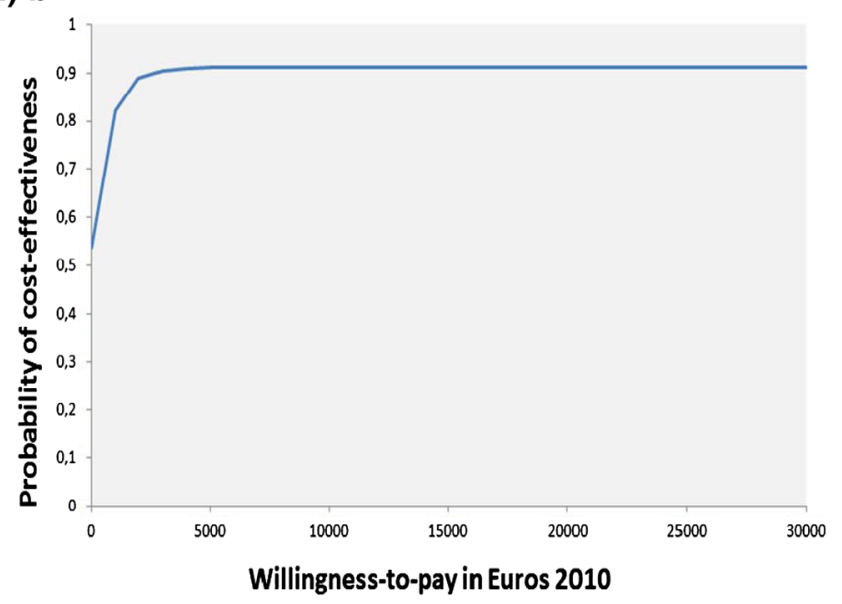

therapy being cost-effective in comparison with physical therapy for different values $(€)$ of willingness-to-pay (2) for perceived recovery (a) and functional status (NDI-DV: continuous outcome) (b)

described by Cyriax, Kaltenborn, Maitland, and Mennel [6]. Second, the participants' baseline functional status scores were relatively favourable, leaving only a small margin for improvement. This reasoning is underscored by a post hoc analysis, indicating that a larger mean difference in functional status (continuous) was found between the MTU and PT group when participants with a mild or no disability (i.e., NDI-DV $<15$ ) were excluded from the analyses ( $\beta-1.83$; $95 \% \mathrm{CI}:-5.44-1.78 ; n=52)$ [10]. Third, MTU was only compared to PT, whereas recent evidence indicates that a combination of manual therapy and PT is most likely to be effective [19]. As economic evaluations are sparse in the field of manual therapy [20] and evidence regarding the relative cost-effectiveness of a combination of manual therapy and PT versus an alternative strategy is lacking, future research into this topic is warranted. 


\section{Strengths and limitations}

A first strength of this study is its pragmatic RCT design. Such a design is acknowledged as the best vehicle for economic evaluations, as its pragmatic approach improves the findings' generalisability to daily clinical practice, whereas the randomisation of participants reduces the possible influence of selection bias [13]. A second strength is that cost-effectiveness was analysed using SUR analyses, which made it possible to correct for the possible correlation between costs and effects [17]. A third strength is that not only QALYs and functional status were used as an outcome measure in the economic evaluation, but also perceived recovery as measured by the GPE. This is important because the GPE was found to capture chronic neck pain patient perceptions of chance in domains that may not be captured by other outcome instruments [21].

The present study also has some limitations. First, cost and effect measure values were collected using self-report, which may have caused "social desirability" and/or "recall bias". Nonetheless, as it seems unlikely that the extent of impairment in recall and/or the degree to which participants gave social desirable answers systematically differed between groups, we do not expect that our reliance on them severely biased the results. Second, even though reduced on-the-job productivity (i.e., presenteeism) was found to account for the biggest share of productivity-related costs due to pain complaints [22], presenteeism costs were not included. This may have resulted in an underestimation of the societal costs. Another limitation concerns the amount of missing data. To deal with this limitation, missing cost and effect data were multiply imputed. Multiple imputation is currently considered the most appropriate method for imputing cost data, as it accounts for the uncertainty about the missing data by creating several imputed data sets [16]. Also, some significant differences in baseline characteristics were observed between patients with complete and incomplete data in both treatment groups, indicating that the complete-case analysis is likely biased by self-selection of participants.

\section{Conclusion}

From a societal perspective, MTU was not cost-effective compared with PT among sub-acute and chronic nonspecific neck pain patients for perceived recovery, functional status, and QALYs. As no clear total societal cost and effect differences were found between MTU and PT, the decision about what intervention to administer, reimburse, and/or implement can be based on the preferences of the patient and the decision-maker at hand.

\section{Compliance with ethical standards}

Funding This study was funded in-part by the Health Care Foundation 'Spaarneland', which is a program for funding innovative projects of one of the health insurance companies in the Netherlands ('Zilverenkruis/Achmea'), while the majority of the study was selffinanced. The Foundation had no further role in the design of the study, in the collection, analysis and interpretation of the data, and in drafting the manuscript.

Conflict of interest RG has a private practice for MTU. There are no competing interests.

Open Access This article is distributed under the terms of the Creative Commons Attribution 4.0 International License (http://crea tivecommons.org/licenses/by/4.0/), which permits unrestricted use, distribution, and reproduction in any medium, provided you give appropriate credit to the original author(s) and the source, provide a link to the Creative Commons license, and indicate if changes were made.

\section{References}

1. Binder A (2006) Neck pain. Clin Evid 15:1654-1675

2. Vos T, Flaxman AD, Naghavi M et al (2010) Years lived with disability (YLDs) for 1160 sequelae of 289 diseases and injuries 1990-2010: a systematic analysis for the Global Burden of Disease Study. Lancet 380(9859):2163-2196

3. Borghouts J, Koes B, Vondeling H et al (1996) Cost-of-illness of neck pain in The Netherlands in 1996. Pain 80(3):629-636

4. Driessen MT, Lin CW, van Tulder MW (2012) Cost-effectiveness of conservative treatments for neck pain: a systematic review on economic evaluations. Eur Spine J 21(8):1441-1450

5. Vos C, Verhagen A, Passchier J et al (2007) Management of acute neck pain in general practice: a prospective study. $\mathrm{Br} \mathrm{J}$ Gener Pract 57(534):23-28

6. Korthals-de Bos I, Hoving J, van Tulder M et al (2003) Cost effectiveness of physiotherapy, manual therapy, and general practitioner care for neck pain: economic evaluation alongside a randomised controlled trial. BMJ 326(7395):911

7. Bosmans JE, Pool JJM, de Vet HCW et al (2011) Is behavioral graded activity cost-effective in comparison with manual therapy for patients with subacute neck pain?: an economic evaluation alongside a randomized clinical trial. Spine 36(18):E1179-E1186

8. Groeneweg R, Kropman H, Leopold H et al (2010) The effectiveness and cost-evaluation of manual therapy and physical therapy in patients with sub-acute and chronic non-specific neck pain. Rationale and design of a Randomized Controlled Trial (RCT). BMC Musculoskelet Disord 11(1):14

9. Kamper SJ, Ostelo RW, Knol DL et al (2010) Global Perceived Effect scales provided reliable assessments of health transition in people with musculoskeletal disorders, but ratings are strongly influenced by current status. J Clin Epidemiol 63(7):760-766 (e761)

10. Jorritsma W, de Vries GE, Dijkstra PU et al (2012) Neck pain and disability scale and neck disability index: validity of Dutch language versions. Eur Spine J 21(1):93-100

11. Brazier J, Roberts J, Deverill M (2002) The estimation of a preference-based measure of health from the SF-36. J Health Econ 21(2):271-292

12. Statistics Netherlands (2014) Consumer price indices. http:// www.cbs.nl/nl-NL/menu/cijfers/kerncijfers/default.htm. Accessed 25 June 2014 
13. Drummond MF, Sculpher MJ, Torrance GW et al (2005) Methods for the economic evaluation of health care programmes. Oxford University Press, New York

14. Hakkaart- van Roijen L, Tan S, Bouwmans C (2010) Handleiding voor kostenonderzoek. Methoden en standaard kostprijzen voor economische evaluaties in de gezondheidszorg. Geactualiseerde versie 2010. CVZ

15. Z-Index. (2009) G-Standaard. http://www.z-index.nl/zorgverl eners. Accessed 25 June 2014

16. White IR, Royston P, Wood AM (2011) Multiple imputation using chained equations: issues and guidance for practice. Stat Med 30(4):377-399

17. Willan AR, Briggs AH, Hoch JS (2004) Regression methods for covariate adjustment and subgroup analysis for non-censored cost-effectiveness data. Health Econ 13(5):461-475

18. Lamers L, Stalmeier P, McDonnell J et al (2005) Measuring the quality of life in economic evaluations: the Dutch EQ-5D tariff. Ned Tijdschr Geneeskd 149:1574-1578
19. (2013) Neck Pain: combining exercise and manual therapy for your neck and upper back leads to quicker reductions in pain. J Orthop Sports Phys Ther 43(3):128-128

20. Gross A, Langevin P, Burnie SJ et al (2015) Manipulation and mobilisation for neck pain contrasted against an inactive control or another active treatment. Cochrane Database Syst Rev 61(3):106-116

21. Evans R, Bronfort G, Mailers M et al (2014) "I know it's changed": a mixed-methods study of the meaning of global perceived effect in chronic neck pain patients. Eur Spine J 23(4):888-897

22. Stewart WF, Ricci JA, Chee E et al (2009) Lost productive time and cost due to common pain conditions in the us workforce. JAMA 290(18):2443-2454 